\title{
Road Selection Using Multicriteria Fusion for the Road-Matching Problem
}

\author{
Maan El Badaoui El Najjar and Philippe Bonnifait, Member, IEEE
}

\begin{abstract}
This paper presents a road selection strategy for novel road-matching methods that are designed to support realtime navigational features within Advanced Driving-Assistance Systems (ADAS). Selecting the most likely segment(s) is a crucial issue for the road-matching problem. The selection strategy merges several criteria using Belief theory. Particular attention is given to the development of belief functions from measurements and estimations of relative distances, headings, and velocities. Experimental results using data from antilock brake system sensors, the differential Global Positioning System receiver, and the accurate digital roadmap illustrate the performances of this approach, particularly in ambiguous situations.
\end{abstract}

Index Terms-Belief theory, Geographical Information System (GIS), Global Positioning System (GPS), localization, sensor fusion.

\section{INTRODUCTION}

I NTELLIGENT autonomous vehicles currently hold the attention of many researchers because they can provide solutions in many applications related to intelligent transportation systems. One example of such a system is the transport of passengers in urban environments using a CyCab [16]. For navigational needs, the vehicle first needs to know its position on the road network and then to retrieve attributes from the appropriate databases. Examples of attributes are maximum authorized speed, width of the road, presence of landmarks for precise localization, etc. Unfortunately, the precise localization on a map cannot be guaranteed because there will often be errors in the estimation of position arising from sensor imprecision and because the map represents a deformed view of the real world: Roads are represented by points-nodes and shaping points - that describe the geometry of the center line.

Vehicle localization on a map has two meanings in the literature of this domain. In many works [2], [11], [13], [18], [19], [25], it refers to the projection of the absolute position estimate onto a segment of the road network stored in the database. In

Manuscript received July 15, 2004; revised July 11, 2005, February 7, 2006, August 30, 2006, and December 8, 2006. This work was carried out in the framework of the French DIVA SAACAM project. The Associate Editor for this paper was Y. Zhao.

M. E. B. El Najjar is with the LAGIS Laboratory, UMR CNRS 8146, Ecole Centrale de Lyon, Université des Sciences et Technologies de Lille, 59655 Villeneuve d'Ascq, France, and he is also a member of the MAIA project of the LORIA-INRIA Lorraine UMR 7503 (e-mail: Maan.E-elnaijar@univ-lille1.fr).

P. Bonnifait is with the Laboratoire Heudiasyc, UMR CNRS 6599, Departement Genie Informatique, Universite de Technologie de Compiegne, 60205 Compiegne, France (e-mail: Philippe.Bonnifait@hds.utc.fr).

Color versions of one or more of the figures in this paper are available online at http://ieeexplore.ieee.org.

Digital Object Identifier 10.1109/TITS.2007.895312 this case, the vehicle is localized when the curvilinear abscissa along the segment is known from the starting node. These "arcmatching" methods therefore introduce geometric distortions since the model of the world is a set of segments, usually with a 10-m absolute error and a 1-m relative error. Alternatively, vehicle localization can refer to absolute localization in the map reference frame. In this case, the localization of the vehicle does not need a projection onto the segments representing the road in the database. Absolute localization can be very useful for the following reasons: In several kinds of databases, including those of the National French Institute of Geography (IGN), attributes, instead of being attached to the arcs representing the roads, can be stored in the database as point objects with an absolute position. Moreover, it is imprecise to suppose that the real trajectory of the vehicle can be modeled by linear arcs. The distortion introduced by such an assumption is amplified if the network database is not accurate. In reality, roads have a nonnegligible width and define areas (and not lines) within which the vehicle can navigate. Furthermore, arc-matching methods are not adapted to the automatic guidance control of vehicles since lateral variation is not observable: Only longitudinal control is possible using speed values attached to the arcs.

The approach presented in this paper is an absolute localization method. The global positions provided by a Global Positioning System (GPS) receiver are converted into the map frame and not projected onto the segments. Therefore, the key issue is the selection of the most likely road from the database, which hereafter is referred to as "road matching." The fusion of the selected segment with the estimated pose is not detailed in this paper. It can be performed recursively by using Kalman filtering, as done in [8], when there is no ambiguity.

Generally, road selection first involves applying a filter that selects all the segments close to the estimated position of the vehicle. The goal is then to select the most likely segment(s) from this subset. Nowadays, since the geometry of roadmaps is more and more detailed, the number of segments representing roads is increasing. The road selection module is an important stage in the vehicle localization process because the robustness of the localization depends mainly on this stage. The road selection stage is also important because it reduces the number of roads to be processed, which is essential for a real-time implementation. To be focused on this point, an accurate map (Géoroute V2) that was provided by the IGN was used in this paper. Its absolute precision is estimated to be several meters in detailed city format. The selection strategy proposed is based on the merging of several criteria using distance, direction, 
and velocity measurements within the framework of Belief theory.

To develop such an approach, it is important to estimate continuously the pose-position and heading — of the vehicle in the frame of the map using GPS because of its affordability and convenience. However, GPS suffers from satellite outages occurring in urban environments, under bridges, through tunnels, or in forests. GPS can thus be seen as an intermittently available positioning system that needs to be backed up by a dead-reckoning system [1]. In this paper, a low-cost odometric method based on the use of encoders attached to the rear wheels is proposed. A dead-reckoned estimated pose is obtained by integrating the elementary rotations of the wheels starting from a given pose. The multisensor fusion of GPS and odometry is performed by an extended Kalman filter (EKF). This kind of formalism is also useful in quantifying the imprecision associated with each estimated pose. As we show in this paper, this information is of particular relevance when quantifying the selection criteria.

The outline is as follows: In Section II, the EKF formalism that is used in performing the sensor fusion of differential GPS (DGPS) and odometry is described. The fusion provides a continuous estimation of the heading of the vehicle and quantifies the estimation error regarding the pose: These two quantities are key parameters for the road selection problem. Next, the architecture of a usual roadmap-matching algorithm is laid out. The road selection method is presented in Section III. The proposed strategy fuses two criteria using Belief theory with a fuzzy representation of sensor information. The final section is the experimental analysis of several tests that are carried out with our experimental car.

\section{Architecture of the RoAD-Matching Strategy}

At present, there would seem to be no ideal solution to the road-matching problem. All the methods that have been developed have their advantages and drawbacks, and they were optimized for the particular application for which they were designed [22], [25]. For the needs of turn-by-turn navigation systems, many of them would appear adequate. However, safety applications that are dedicated to Advanced Driving-Assistance Systems (ADAS) require the ability to estimate the confidence in the road-matched positions. If the confidence is too low, the information that is retrieved from the Geographical Information System (GIS) will not be used.

Arc-matching methods consist of localizing the vehicle on the road network modeled by linear arcs. Several approaches have been proposed in the literature. Geometric approaches [2], [9], [11], [13] rely on the correlation of the estimated trajectory with the shape of the road network. Fuzzy techniques [14], [21] use models and rules to take into account map and sensor errors. These techniques are able to detect off-road situations. In the framework of Bayesian filtering, Kalman approaches [3], [15], [26] and particle filter [10] techniques have been developed. The matching relies in this case on a map posetracking paradigm in which the map data are treated as an observation. The ability to characterize several solutions in ambiguous situations is a new preoccupation that can be formu-

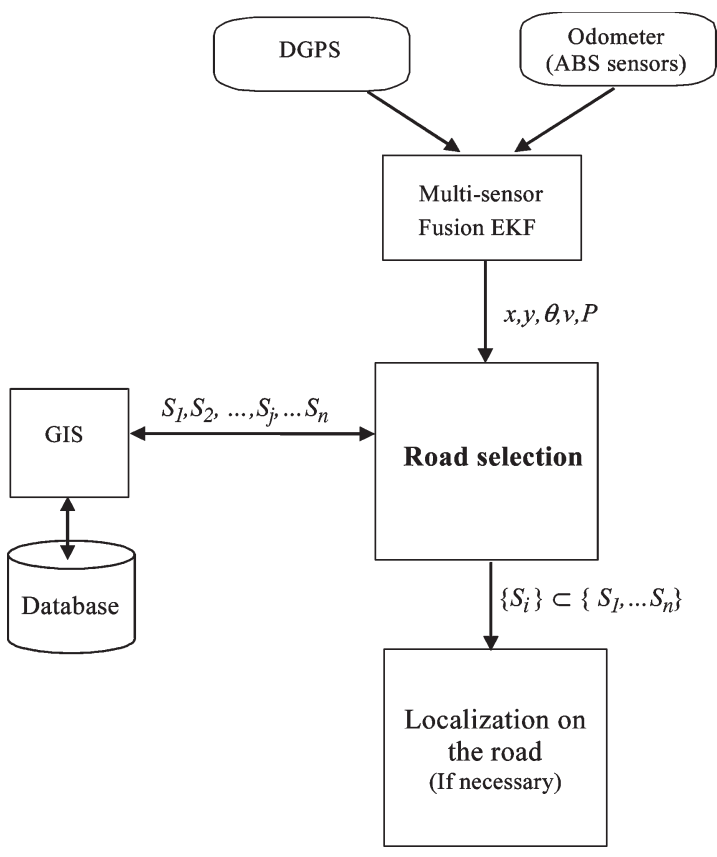

Fig. 1. Schematic diagram of the proposed road-matching algorithm.

lated with a multihypothesis scheme [17] or with a multimodal density probability [10].

In this paper, we propose a new formulation to solve the problem. Given an estimated absolute localization with high availability in the frame of the map, the road matching is reduced to the selection of the most likely segment of the database. According to this approach, there is no projection on the segments representing the roads. If a projection is needed to retrieve the attributes that are attached to the segment, this projection is performed afterward. The key issue is to have a good estimate of the pose error for the fusion process. Obviously, the better the estimation of the pose, the less ambiguous the selection process.

The road selection process can be described as in Fig. 1, where $v$ denotes the mean speed of the rear wheels. Multisensor fusion gives an estimation of the pose $X=(x, y, \theta)^{t}$ with its associated covariance matrix $P$. The question is then to select the most likely segment(s) using a GIS. To accelerate processing (a map contains thousands of roads, each made up of several segments), an initial filter selects the $n$ road segments $\left\{S_{1}, \ldots, S_{n}\right\}$ that are located within an interest window that is chosen for consideration of real time or reliability. The center of the circle is the estimation of the current position $(x, y)$ of the car. This initial filter can be applied at low frequency and interpreted as a cache memory as in [27]. Next, the road selection stage selects the credible segments. If necessary, the location on the segment can be done by projection. This final stage is usually called map matching.

\section{A. Road Selection}

This problem consists of selecting the "good" segments from the subset $\left\{S_{1}, \ldots, S_{n}\right\}$. This is also called the road reduction filter problem in [8] and [23]. 


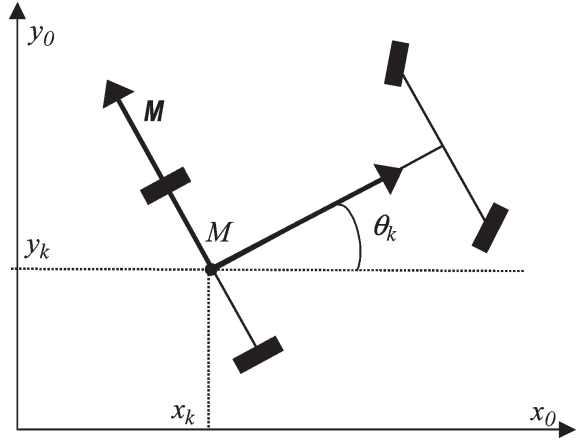

Fig. 2. Mobile frame attached to the car.

This stage is difficult because of several factors.

- The position is estimated with errors that can be magnified by multipath effects. In addition, the transformation between the GPS coordinates (World Geodetic System 1984 reference system) and the projection frame of the map [here, the French Nouvelle Triangulation Française (NTF) Lambert coordinate system] can induce errors.

- The coordinates of the segments are falsified by errors due to terrain measurements that are carried out by cartographers as well as due to numerical approximation.

- The road network in the database does not always correspond to reality, i.e., it can contain old roads that no longer exist or new roads that are not yet in the database.

- The map does not contain all road network details. For example, a roundabout can be represented as a simple point.

- The vehicle is moving on a 3-D surface, whereas the map represents a planar view.

- The vehicle does not travel exactly on the segments representing the roads.

Section III will present a strategy using Belief theory.

\section{B. GPS and Antilock Brake System (ABS) Fusion}

High availability positioning is a basic necessity in the roadmatching process. As GPS is subject to satellite outages, we propose to use the ABS sensors of the rear wheels of a car to provide a continuous positioning. As most modern cars have an ABS system, it is a very cheap solution that does not require additional sensors. In this section, the fusion of GPS and odometry with an EKF is described.

Here, we consider a car-like vehicle with front-wheel drive. The mobile frame is chosen with its origin $M$ attached to the center of the rear axle. The $x$-axis is aligned with the longitudinal axis of the car (see Fig. 2).

At time index $t_{k}$, the vehicle position is represented by the $\left(x_{k}, y_{k}\right)$ Cartesian coordinates of $M$ in a world frame. The heading angle is denoted as $\theta_{k}$. If the road is perfectly planar and horizontal and if the motion is locally circular, the evolution model is expressed as [4]

$$
\left\{\begin{array}{l}
x_{k+1}=x_{k}+\Delta_{k} \cdot \cos \left(\theta_{k}+\frac{\omega_{k}}{2}\right) \\
y_{k+1}=y_{k}+\Delta_{k} \cdot \sin \left(\theta_{k}+\frac{\omega_{k}}{2}\right) \\
\theta_{k+1}=\theta_{k}+\omega_{k}
\end{array}\right.
$$

where $\Delta_{k}$ is the length of the circular arc that is followed by $M$, and $\omega_{k}$ is the elementary rotation of the mobile frame. These values are computed using the ABS measurements of the rear wheels. In this formulation, the values of the scale factors of the ABS sensors are supposed to be precisely known after a calibration stage.

Let us denote $u_{k}=\left[\Delta_{k}, \omega_{k}\right]^{t}$ and $X_{k}=\left[x_{k}, y_{k}, \theta_{k}\right]^{t}, \alpha_{k}, \gamma_{k}$ as the model noise and the ABS noise, respectively. Equation (1) can be rewritten as

$$
X_{k+1}=f\left(X_{k}, u_{k}, \gamma_{k}\right)+\alpha_{k} .
$$

When a GPS fix is available, it is first converted from geographical coordinates into Cartesian coordinates $Y_{k}$ by a projection in the frame of the map (here, French Lambert I). Then, a correction of the odometric estimate is performed using an EKF, due to the following observation model:

$$
Y_{k}=\left[\begin{array}{c}
x_{\mathrm{gps}, k} \\
y_{\mathrm{gps}, k}
\end{array}\right]=\left(\begin{array}{ccc}
1 & 0 & 0 \\
0 & 1 & 0
\end{array}\right)\left(\begin{array}{l}
x_{k} \\
y_{k} \\
\theta_{k}
\end{array}\right)+\beta_{k} .
$$

$\beta_{k}$ denotes the GPS measurement noise. The covariance matrix $Q_{\beta}$ can be estimated in real time using the National Marine Electronics Association sentence "GNSS error STatistics (GST)," which was provided by the Trimble AgGPS132 receiver that was used in the experiments. Therefore, the noise $\beta_{k}$ is not stationary. It will be noticed that a DGPS receiver (whose precision is well adapted to the use of a precise roadmap) was used.

This architecture can be seen as a "loosely coupled fusion system." The heading $\theta$ is not directly measured since the GPS is used as a position sensor. Let us study its observability.

From (1), we have

$$
x_{k+1}=x_{k}+\Delta_{k} \cdot \cos \left(\theta_{k}+\frac{\omega_{k}}{2}\right) \quad \text { and } \quad \theta_{k+1}=\theta_{k}+\omega_{k} \text {. }
$$

Then

$\theta_{k}=\theta_{k+1}-\omega_{k} \quad$ and $\quad x_{k+1}=x_{k}+\Delta_{k} \cdot \cos \left(\theta_{k+1}-\frac{\omega_{k}}{2}\right)$.

By making a change of index, the heading $\theta_{k}$ can be expressed as

$$
\theta_{k}=\operatorname{arcos}\left(\frac{\mathrm{x}_{\mathrm{k}}-\mathrm{x}_{\mathrm{k}-1}}{\Delta_{\mathrm{k}-1}}\right)+\frac{\omega_{\mathrm{k}-1}}{2} .
$$

Therefore, the heading is expressed as a combination of $\left(x_{k}, x_{k-1}, \Delta_{k-1}, \omega_{k-1}\right)$. Since $x$ is observable (directly measured by the GPS), $\theta$ is also observable. Nevertheless, the mathematical expression is not defined when the speed is zero, which demonstrates that the heading is observable when the vehicle is moving. When the vehicle is motionless, the heading is not observable and does not need to be estimated.

The EKF follows a predictor/estimator paradigm at a frequency of $1 \mathrm{~Hz}$ using the pulse-per-second signal. The prediction stage is computed using the ABS measurements if there is a displacement of the vehicle. This guarantees that the EKF correctly reconstructs the heading angle since its observable. 


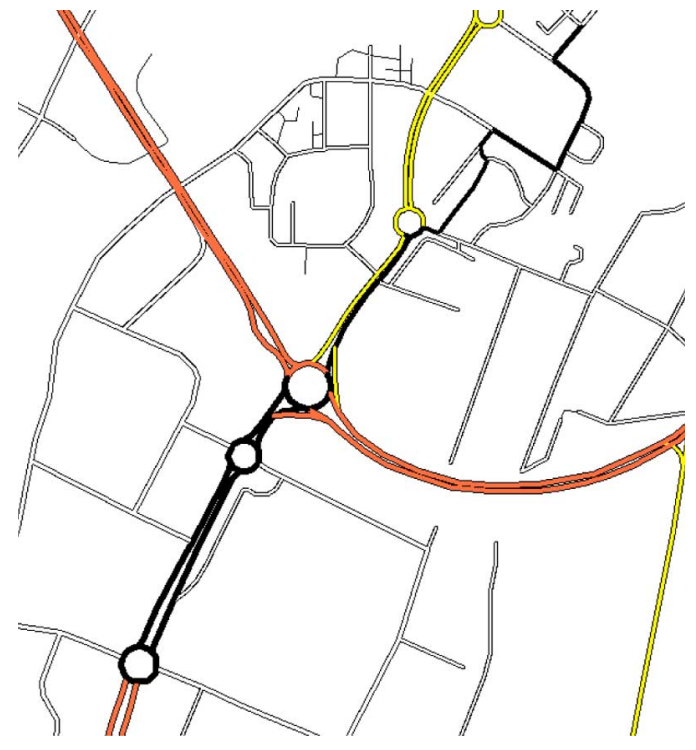

Fig. 3. Top view of the experimental run (in black).

Then, in the estimation stage, the GPS corrects the drift of the dead-reckoned pose. To use good GPS fixes with a good precision, the GPS receiver has to be tuned with very selective masks (dilution of precision, elevation, and signal/noise ratio). For this reason, the GPS data are intermittent, but one can give a high belief in this information when it is present.

The equations are not described here, but details can be found in [8]. Hereafter, we report the results of a test performed over a $4.5-\mathrm{km}$ route (see Fig. 3 ).

The behavior of the filter can be characterized by examining the $x$ and $y$ innovations (differences between the DGPS measurements and the predicted measurements). It can be seen from Fig. 4 that the $x$ innovation is zero mean. This figure also shows the probability distribution of the $x$ innovation errors, which confirms its zero-mean behavior. Moreover, the autocorrelation is close to white noise since the sampling period equals $1 \mathrm{~s}$. This global behavior indicates that the filter is correctly tuned.

In the framework of the Action de Recherche pour une COnduite Sécurisée (ARCOS) French research program, this system was validated in relation to a centimeter-level accuracy postprocessed kinematic GPS system. The horizontal precision is on the order of $2 \mathrm{~m}(1-\sigma)$ when the GPS employs a differential pseudorange correction [12].

Finally, using the odometric model, the EKF estimates continuously the $X$ vector and the covariance matrix $P$ of the estimated error, even when the satellite signal is blocked by bridges, tunnels, buildings, etc. In such a situation, the pose is predicted due to (1) and the ABS sensors. In this case, the drift depends only on the traveled distance and not on time. It has been estimated to $10 \%$ of the traveled distance, which is sufficient for short GPS outages. Fig. 5 illustrates the lateral drift during small GPS outages. The $3 \sigma$ bound proves the consistency of the filter.

\section{RoAd SElection Using Multicriteria Fusion}

Intuitively, taking many factors into consideration makes it possible to avoid matching errors that easily result from a single point-of-view selection. Therefore, to improve map-matching methods, different information sources have to be merged to provide better selection criteria for deciding the best match. For example, Quddus et al. [18] have used the "GPS position relative to the road link" and an "intersection relation between the GPS trace and the road links." Syed and Cannon [21] have used the "average distance traveled on current link" and the "large distance traveled on current road link." In addition, if the base road network contains detailed road attributes (speed limits, one-way lanes, etc.) that potentially restrict a certain routing behavior, they can potentially be utilized to further filter inappropriate road links.

Current trends in map-matching development have begun to incorporate probabilistic and fuzzy elements that are more tolerant with uncertainty (confidence in given information), imprecision (noises), and model approximations. Uncertainty typically exists in the database street map.

Several approaches can be followed to fuse multidimensional selection criteria. The commonly used one combines selection factors with a weighting scheme. The weighting factors are typically derived empirically from data testing or from adaptive-fuzzy-network-based training [14]. A different approach can be implemented in the framework of Belief theory, which allows partial knowledge to be taken into account. The Dempster-Shafer rule can be used to fuse the criteria. Moreover, because of its commutativity and associativity, it is possible to incorporate any criterion and to fuse it in any order. Belief theory also facilitates the estimation of the degree of belief in a selected road. The detection of off-road travels, for instance, is characterized by the fact that all the segments have a low belief.

The following first presents the concepts used in Belief theory. The selection criteria will next be described, and finally, the fusion of these criteria will be illustrated by a real example.

\section{A. Belief Theory Concepts}

Belief theory admits uncertainty into the reasoning process and suggests a way for combining uncertain data. This theory was introduced by Dempster [5] and mathematically formalized by Shafer in 1976 [20]. It is the generalization of Bayesian theory in the treatment of uncertainty. Generally, this theory is used in a multisensor context to fuse heterogeneous information to obtain the best decision.

The basic entity is a set of all possible answers (also called hypotheses) to a specific question. This set is called the frame of discernment and is denoted as $\Theta$. All the hypotheses must be exclusive and exhaustive, and each subset of the frame of discernment can be a possible answer to the question. The degree of belief of each hypothesis is represented by a real number in $[0,1]$ that is called mass function $m(\cdot)$. It satisfies the following rules:

$$
\begin{aligned}
m(\phi) & =0 \\
\sum_{A \subseteq \Theta} m(A) & =1 .
\end{aligned}
$$



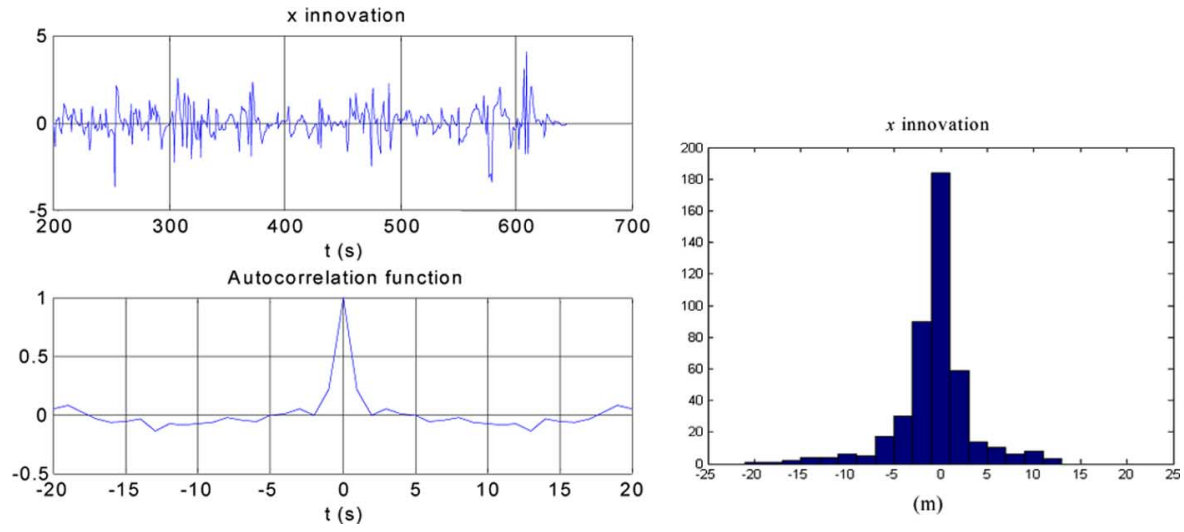

(m)

Fig. 4. Autocorrelation and distribution of the $x$ innovation of the EKF.

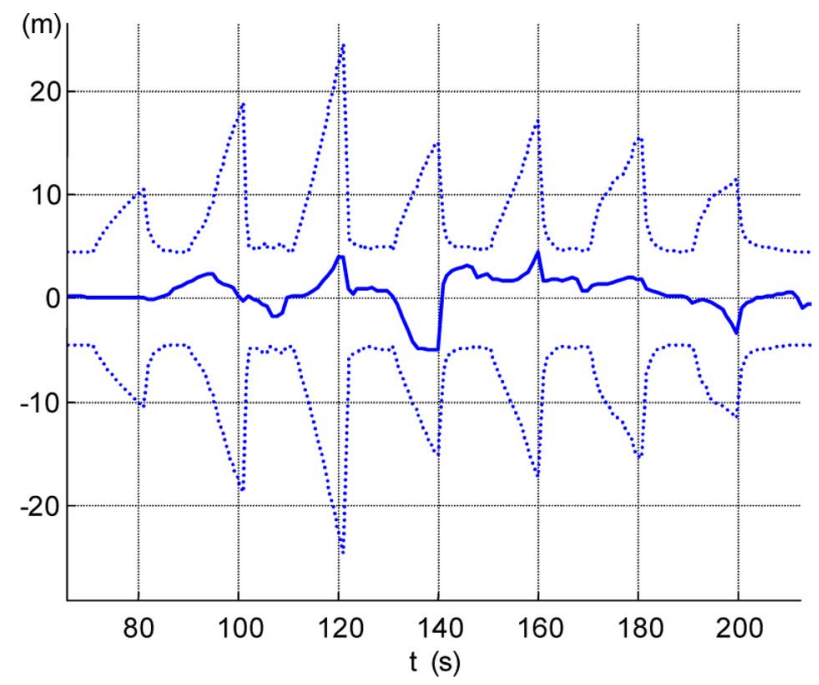

Fig. 5. Drift of ABS-only localization during small GPS outages.

A mass function is defined for each different piece of evidence. Each piece of evidence $A$, for which $m(A) \neq 0$, is called a focal element. The notion of mass is very close to the notion of probabilistic mass, except that it is not limited to a single hypothesis, but it is possible to attribute a mass for a union of hypotheses: This is the main difference with respect to Bayesian theory.

Belief theory requires the affectation of elementary probabilistic masses that are defined on $[0,1]$. The mass assignment is computed on the definition referential $2^{\Theta}$, i.e.,

$$
\begin{aligned}
& 2^{\Theta}=\left\{\oslash, H_{1}, H_{2}, \ldots H_{n}, H_{1} \cup H_{2}, \ldots,\right. \\
&\left.H_{i} \cup H_{j} \cup H_{k} \cup H_{l} \cup \ldots H_{n}\right\} .
\end{aligned}
$$

This distribution is a function of the knowledge about the source. The whole mass that is obtained is called "basic mass assignment." The sum of the component masses is equal to 1. Each expert—also called source of information-defines a mass assignment according to its opinion about the situation.
Associated with each basic assignment, belief $\mathrm{Bel}$ and plausibility $P l$ are defined as follows:

$$
\begin{aligned}
\operatorname{Bel}(A) & =\sum_{B \subseteq A} m(B) \\
\operatorname{Pl}(A) & =\sum_{B \cap A \neq \phi} m(B) .
\end{aligned}
$$

Belief and plausibility are bound by the following relationship:

$$
P l(A)=1-\operatorname{Bel}(\bar{A})
$$

where $\bar{A}$ denotes the complement of $A$.

\section{B. Application to the Road Selection}

We consider ADAS applications that are related to road safety, for instance, curve warning systems that inform the driver that he is going too fast while approaching a curve. Therefore, only geometrical criteria are used because they are not influenced by human errors. This means that a criterion such as the speed of the vehicle is in accordance with the speed limit is not considered.

The two criteria that are chosen in this paper can be formulated as follows.

1) The vehicle location is close to a segment of the neighborhood. This criterion depends on the error ellipse.

2) The segments on which the vehicle can be located are those that have an angle close to the heading of the vehicle. This criterion depends on the estimated $3 \sigma$ bound of the heading and on the speed of the car.

To build mass assignments, the inaccuracy of the various information sources (GPS, odometer, and digital map) is considered, and certain physical observations, for instance, that a car with a speed of $40 \mathrm{~m} / \mathrm{s}$ cannot be orthogonal to the direction of the segment, are modeled. With this approach, information sources (i.e., criteria) are worked out from sensor data.

The problem of mass assignment for each criterion can be tackled in a global or a local way. The global strategy involves 


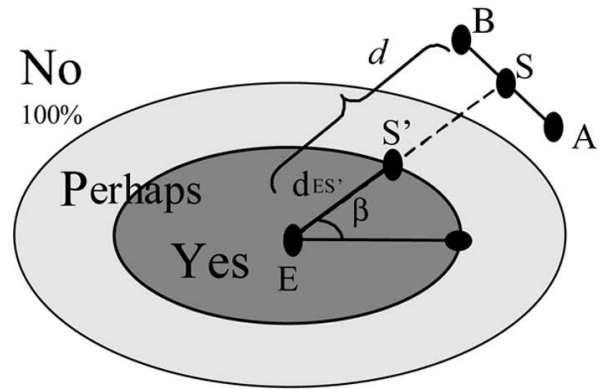

Fig. 6. Case of a noncredible segment.

the simultaneous inspection of all the segments that are selected around an estimated position when affecting the masses. The local strategy treats each segment separately with respect to the criterion under consideration. Both strategies have been studied. We have concluded that the local strategy is more effective, particularly for a real-time application.

The frame of discernment that we use is $\Theta=\{Y e s, N o\}$, corresponding to the answer to the following question: Is this segment the good one? The definition referential is then $2^{\Theta}=\{$ Yes, No, Perhaps $\}$.

1) Proximity Criterion: The proximity criterion is based on the measurement of the Euclidean distance between the estimated position and each segment in the road database. To take into account the estimation error on the position, a Gaussian ellipse is built using the covariance matrix $P$ of the state vector $X[8]$. The probability that a given state $X s$ is included in a $40 \%$ ellipse that is centered on the estimate $X$ is expressed as

$$
\begin{aligned}
& p(X s)=(2 \pi)^{-n / 2} \cdot P^{-1 / 2} \\
& \cdot \exp \left(-\frac{1}{2}(X s-X)^{T} P^{-1}(X s-X)\right) .
\end{aligned}
$$

The equiprobable states $X s$ that characterize a $k \%$ ellipse are given as follows:

$$
(X s-X)^{T} P^{-1}(X s-X)=k^{2} .
$$

The value of $k$ defines the percentage of probability. In two dimensions

$$
p(X s)=1-\exp \left(-\frac{k^{2}}{2}\right) .
$$

For example, $k=1$ implies a $40 \%$ probability that the real position is effectively in the ellipse error. Conversely, given a chosen probability, the corresponding value of $k$ can be computed. This value allows the semiminor and semimajor axes of the ellipse to be determined. For the proximity criterion, the estimated error of the position is quantified by an ellipse of $99 \%$ equiprobability that is produced by the EKF (drawn in dark gray in Fig. 6). The estimated position $E$ is at the center of the ellipse.

To allot a mass to a candidate segment $[A B]$, we proceed as follows: Let $d$ denote the distance between the segment and point $E$.

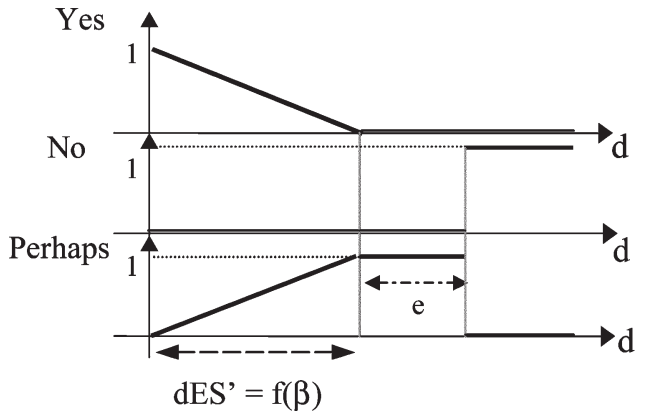

Fig. 7. Mass assignment of the proximity criterion.

Point $S^{\prime}$ falls at the intersection between the segment $[E S]$ and the ellipse. The distance $d E S^{\prime}$ depends on angle $\beta$, which forms segment $\left[E S^{\prime}\right]$ in the ellipse coordinates system. In the zone $d<d E S^{\prime}$, with a fuzzy modeling obtained by a probability-possibility transformation [6], [24], the degree of membership is quantified. The first curve presented in Fig. 7 assigns a mass to the $Y$ es assumption.

In complementing the mass of the $Y$ es hypothesis, the mass to the Perhaps hypothesis is allotted. The mass of Perhaps remains constant (equals to 1 ) for $d E S^{\prime}<d<d E S^{\prime}+e$ to consider the errors $e$ on the segment coordinates of the database. Finally, the mass of the No assumption is a step function starting from the distance $d=d E S^{\prime}+e$.

In conclusion, the mass assignment of the proximity criterion depends on two variables:

1) the distance $d$ between the center of the ellipse and the segment;

2) the angle $\beta$ between the distance support and the major axis of the ellipse.

The problem becomes more complicated when considering the width of the road. We propose modeling the road by a rectangle that is centered on the segment, the length of which is equal to the length of the segment. The exact influence of the width of the road $l$ is difficult to take into account in the computations of the criterion because $l$ modifies the values of $\beta$ and $d$. To simplify, we have chosen the following strategies:

1) if the orthogonal projection of $E$ exists inside segment $[A B], d=$ dortho $-l$ [Fig. 8(a)];

2) if the orthogonal projection of $E$ does not exist inside segment $[A B], d=\min (d 1, d 2, d 3)$ [Fig. 8(b)].

2) Angular Criterion: In this section, a mass-assignment function is proposed to reflect the fact that the most credible segments are those that have an angle close to the heading of the vehicle.

Fig. 9 presents the fuzzy modeling of the absolute value of the difference between the heading of the vehicle (denoted as $\theta$ ) and the heading of the candidate segment (denoted as $\alpha$ ), i.e.,

$\Delta$ Heading $=\min (|\alpha-\theta|,|\alpha-\theta+\pi|)$ with $\theta \in[0, \pi]$. 
(a)

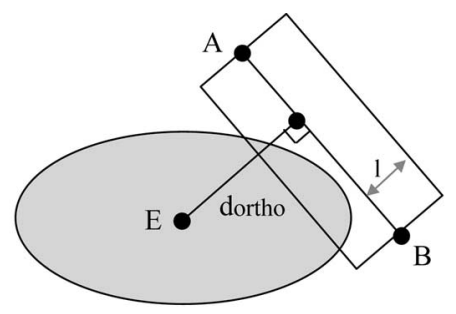

(b)

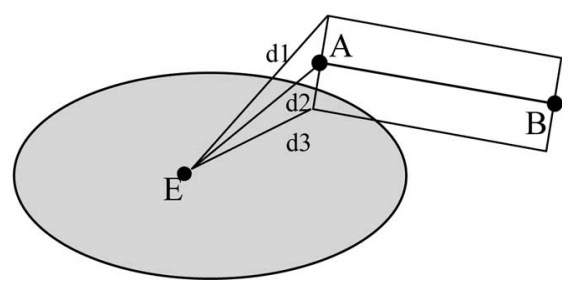

Fig. 8. Computation of the distance $d$ with a rectangle rendering the road.
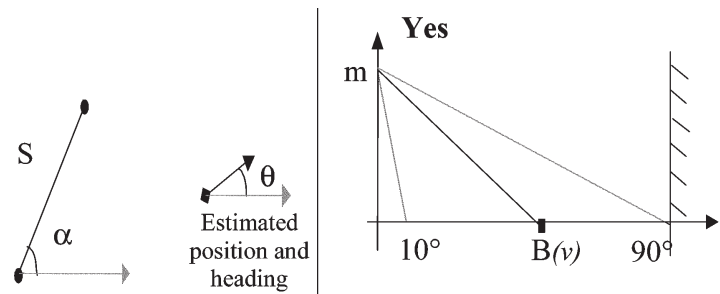

AHeading

Fig. 9. Mass assignment of the $Y$ es hypothesis for the angular criterion. On the left, $S$ represents a candidate segment.

This curve is adaptive and depends on the speed of the vehicle and the standard deviation of the estimation error of the heading angle.

Let $m$ be the maximum belief that can be assigned to the $Y e s$ hypothesis (see Fig. 9). $m$ varies according to $\sigma_{\theta}$ that is estimated by the EKF, i.e.,

$$
m\left(\sigma_{\theta}\right)=\max \left\{1-\frac{6}{\pi} \sigma_{\theta}, 0\right\}
$$

This strategy has been developed to model the fact that an uncertain heading will not induce an important mass to the $Y$ es hypothesis. Let suppose that $3 \sigma_{\theta}=\pi / 2$ represents the situation of the total lack of knowledge on the estimation of the heading. In other words, the real heading is in the interval $[\hat{\theta}-(\pi / 2), \hat{\theta}+(\pi / 2)]$. In this case, the heading criterion must be reduced to the maximum: In (15), the mass assignment to the $Y$ es hypothesis equals 0 .

The scalar value $B$ fixes the angular limit that is tolerated at a given velocity $v$, i.e.,

$$
B(v)=\pi / 2-\lambda \cdot v
$$

where $\lambda=\left(90^{\circ}-10^{\circ}\right) \cdot \pi /\left(V_{\max } \cdot 180^{\circ}\right)$, and $V_{\max }$ is the maximum speed of the vehicle.

Equation (16) is a heuristic model that indicates that if the speed of the vehicle is high, the difference between the heading and the segment direction should be small.
The Perhaps mass assignment is determined by computing the complement of the mass of Yes. The mass of No starts from the limit angle that is tolerated for a given speed $B(v)$ and reaches 1 when the angle is equal to $90^{\circ}$ (Fig. 10).

3) Criteria Fusion: To improve the information that is obtained from two different single sources $S_{1}$ and $S_{2}$, a combination of their mass assignments can be performed according to the Dempster-Shafer rule. Let $A, A_{i}$, and $B_{i}$ be assumptions of the reference frame. The merging of the knowledge of $S_{1}$ and $S_{2}$ is given by

$$
m_{\Theta}(A)=\sum_{A_{i} \cap B_{j}=A} m_{\Theta}^{S_{1}}\left(A_{i}\right) \cdot m_{\Theta}^{S_{2}}\left(B_{j}\right) .
$$

If there are some conjunctions that are not focal elements, a renormalization step is necessary to satisfy the rule $m(\phi)=0$. The coefficient of renormalization is called $k_{\theta}$ and defined as

$$
k_{\theta}=\sum_{A_{i} \cap B_{j}=\phi} m_{\Theta}^{S_{1}}\left(A_{i}\right) m_{\Theta}^{S_{2}}\left(B_{j}\right)
$$

which represents the incoherence between the different sources. If we set $K_{\theta}=1 /\left(1-k_{\theta}\right)$, the normalized expression of the combination is given by

$$
m_{\Theta}(A)=K_{\theta} \cdot \sum_{A_{i} \cap B_{j}=A} m_{\Theta}^{S_{1}}\left(A_{i}\right) \cdot m_{\Theta}^{S_{2}}\left(B_{j}\right)
$$

This combination rule is independent of the order in which pieces of evidence are combined when more than two pieces of evidence are involved.

After the combination step, several decision rules can be used to obtain the final result. It is then possible to adjust a desired behavior. If an optimistic decision is desired, the maximum of plausibility should be used, and conversely, the maximum of belief should be used for a pessimistic decision. Many other decision rules exist in Belief theory, particularly for nonexhaustive frames of discernment. More information about them can be found in [7].

The decision-making strategy adopted here is to retain the most credible segments according the law of ideal decision. The likelihood of a singleton assumption is characterized by its belief and its plausibility, which are calculated using the set of masses. These quantities correspond respectively to the minimum probability and the maximum probability that an assumption is true. Consequently, a law of decision without ambiguity is when an assumption has a belief higher than the plausibility of any other assumption.

The conflict computed in the Dempster-Shafer fusion rule is large when the two criteria are in total confusion. Therefore, we eliminate the segments that present a significant conflict. In our experiments, we used a threshold of 0.5. 


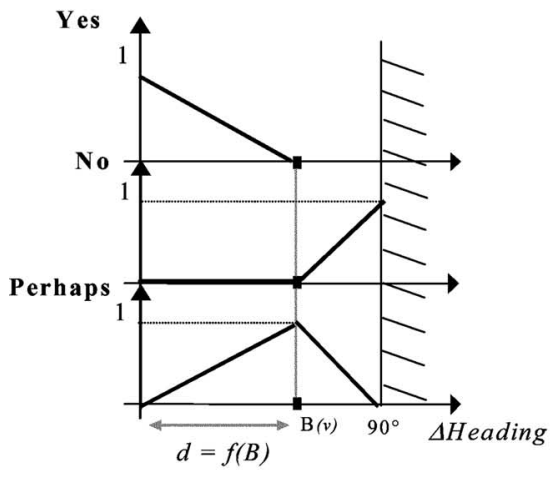

(a)

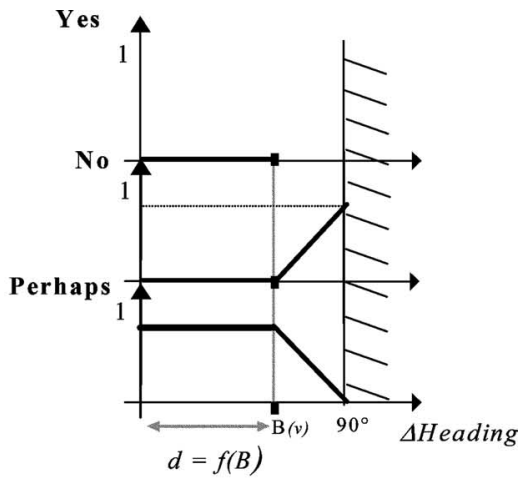

(b)

Fig. 10. Examples of mass assignment at a given velocity. (a) $\sigma_{\theta}=0$. (b) $\sigma_{\theta}=\pi / 2$

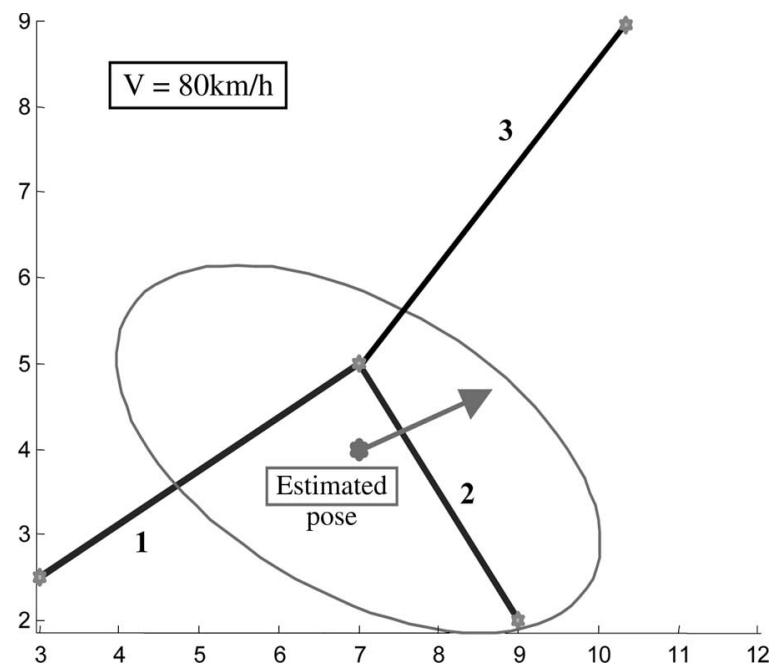

Fig. 11. Estimated position and heading of the vehicle and three candidate segments.

In the following, a segment that satisfies the law of ideal decision with small conflict will be called a credible segment.

\section{Illustrative Example: Approaching a Junction}

Let us take a real case study to illustrate the method. In Fig. 11, the vehicle is traveling on the road that is represented by segments 1 and 3 , at a speed of $80 \mathrm{~km} / \mathrm{h}$. Estimation errors and digital map errors give rise to an erroneous estimated position that is closer to segment 2 than to the others. In the following, the mass attribution, the fusion, and the decision stages are described for each segment.

Figs. 12-14 show the mass assignments that are generated by the belief functions. It will be noticed that for segments 1 and 3 , the proximity and the heading criteria are in agreement since both of them assign a high degree of belief to the $Y e s$ hypothesis, a low degree of belief to the Perhaps hypothesis, and nothing to the No hypothesis. Conversely, segment 2 presents a total conflict between the two criteria.

Fig. 15 shows the results of the fusion of the criteria with the Dempster-Shafer rule without normalization. Notice that segment 2 presents a clear conflict. The fusion of the criteria concerning segments 1 and 3, however, indicates a strong belief on the $Y$ es hypothesis.
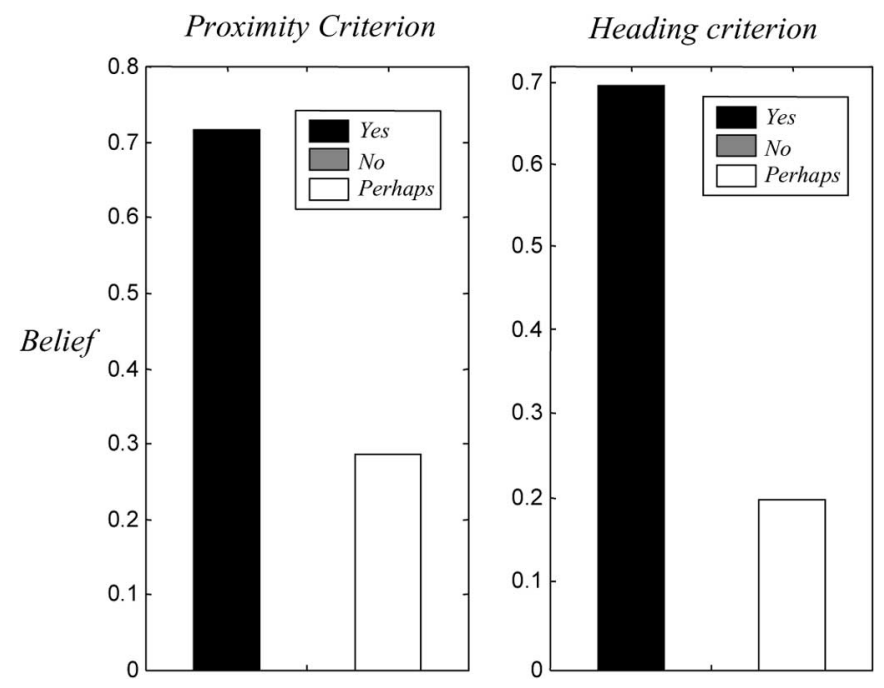

Fig. 12. Mass assignment for segment 1.
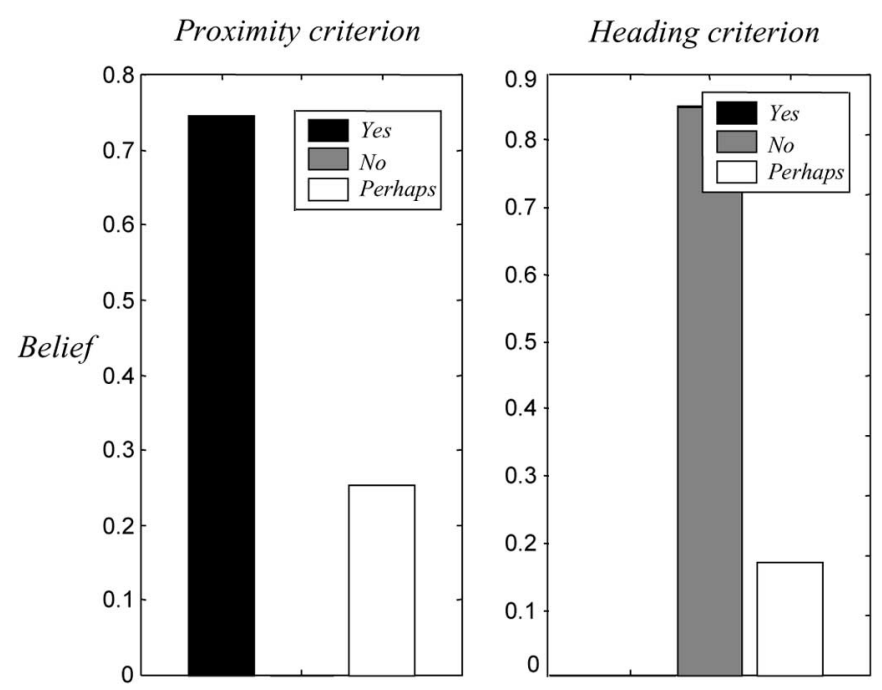

Fig. 13. Mass assignment for segment 2.

To decide if a segment is a good candidate, the conflict generated by the fusion stage is first considered. As it is important for segment 2, this segment is eliminated. Next, the ideal decision law is applied after normalization of the masses. This 

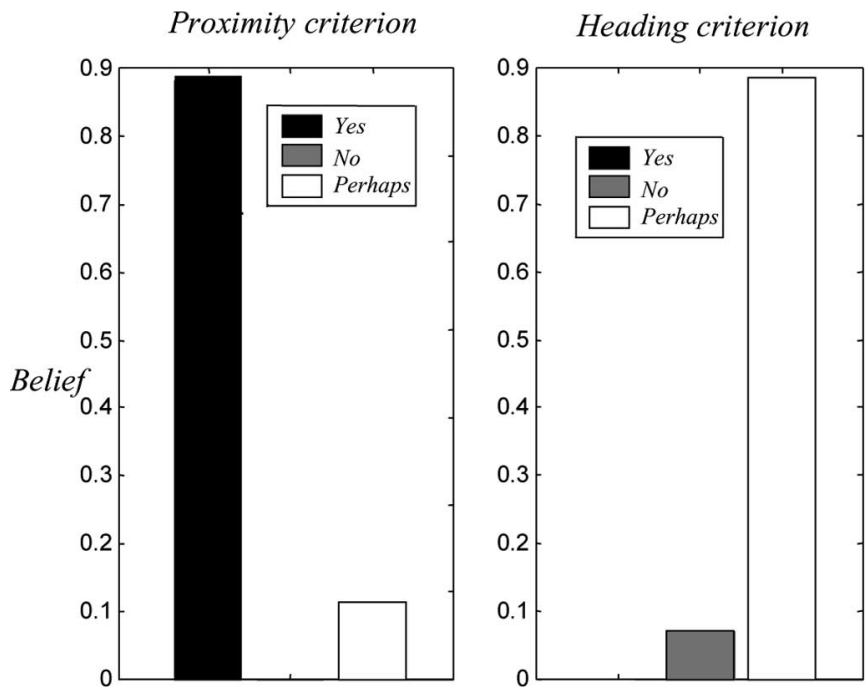

Fig. 14. Mass assignment for segment 3.
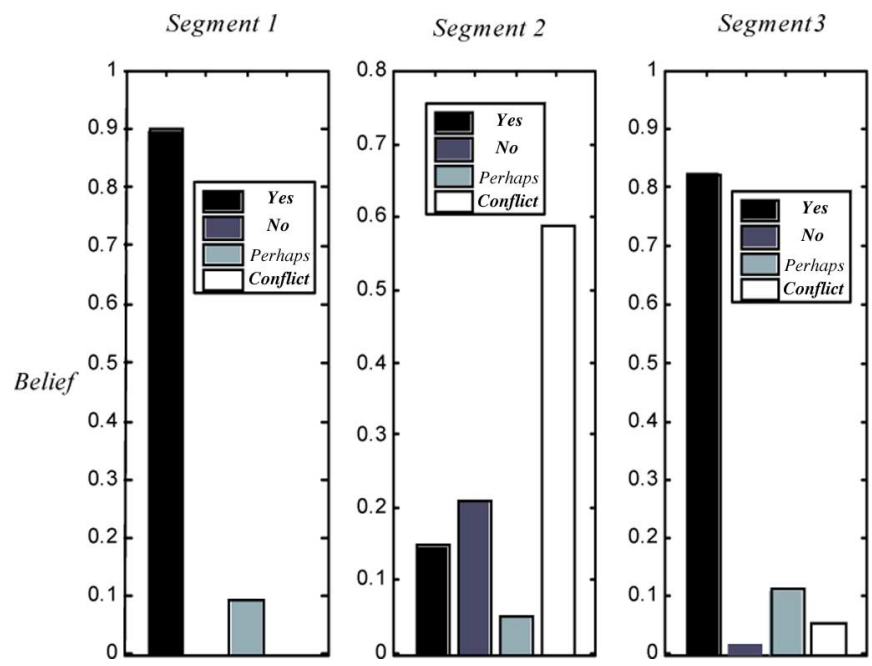

Fig. 15. Fusion results without normalization.

law simply means here that if the belief in the $Y$ es hypothesis is greater than the sum of the No and Perhaps hypotheses, the segment in question is credible. Fig. 16 shows that segments 1 and 3 are selected. This result corresponds to the real situation.

\section{EXPERIMENTAL RESULTS}

The algorithm works in real-time conditions with a frequency of $1 \mathrm{~Hz} .{ }^{1}$ The GPS receiver that was used was a Trimble AgGPS132, L1-only, working with geostationary broadcasted pseudorange corrections (Omnistar).

Fig. 17 presents an overhead view of an experimental test that is performed in Compiègne. The map database is managed and interfaced by the "Geoconcept" GIS software.

To illustrate the road selection method, we will examine how it deals with ambiguous situations. The first situation involves a motorway exit (Fig. 18). This situation is very ambiguous because the angles of three segments (the motorway, the exit

\footnotetext{
${ }^{1}$ Using an Intel Pentium III 700-MHz processor.
}

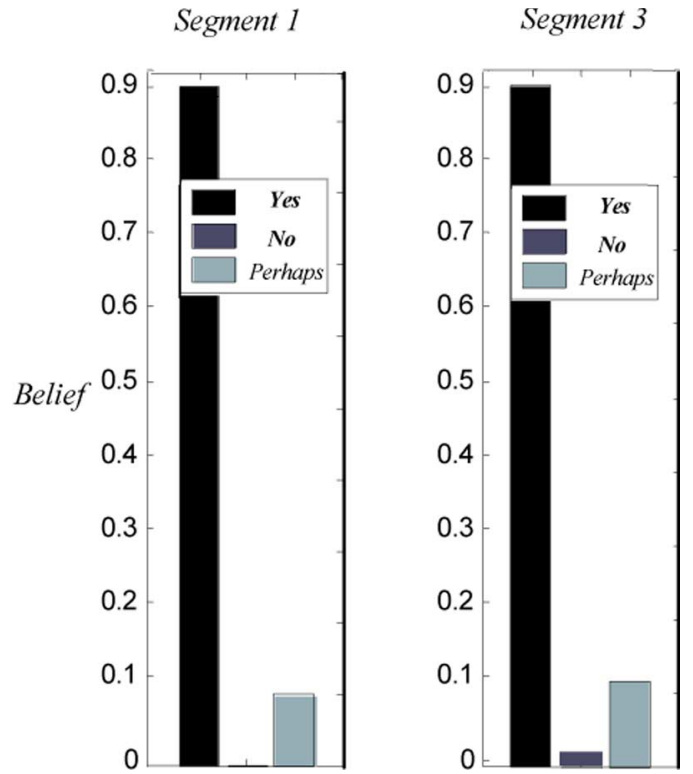

Fig. 16. Fusion results with normalization.

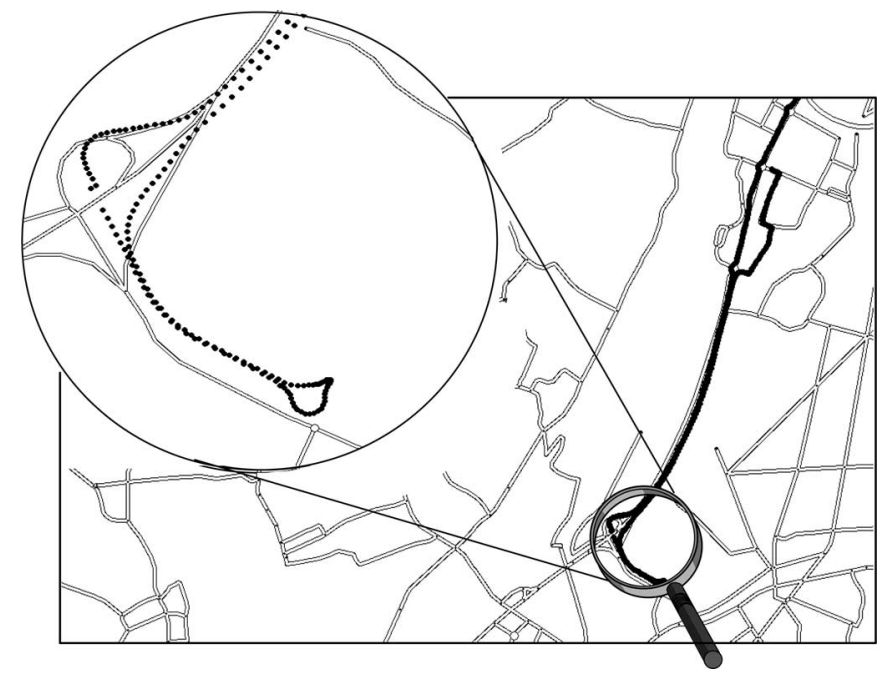

Fig. 17. Experimental situation on the "IGN Géoroute" (estimated positions are dotted).

ramp, and the entrance ramp) are close to the heading of the car. Moreover, they have a common point that is very close to the estimated position.

At the beginning, three segments are selected (in bold in Fig. 18). Two of them correspond to the motorway and one to the exit ramp. As we should expect, the entrance ramp (located on the opposite side of the road) is not selected, owing to the angular criterion. Afterward, the situation is still ambiguous (Fig. 19) until the difference between the car's heading and the angles of the motorway segments becomes significant. Then, the system is able to assert that the car is on the exit ramp (Fig. 20).

To provide a complete view of the evolution of beliefs with respect to the exit ramp, Fig. 21 shows the evolution of belief in selected segments for about ten positions. One can notice that the evolution of belief represents reality well. When several segments are credible, at a given moment, the road 


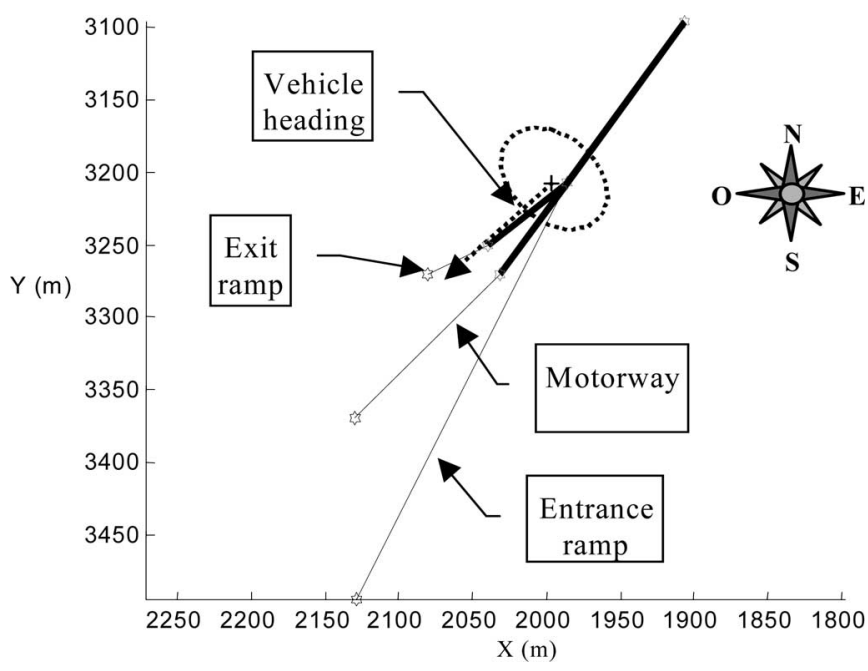

Fig. 18. Car exiting the motorway (local frame).

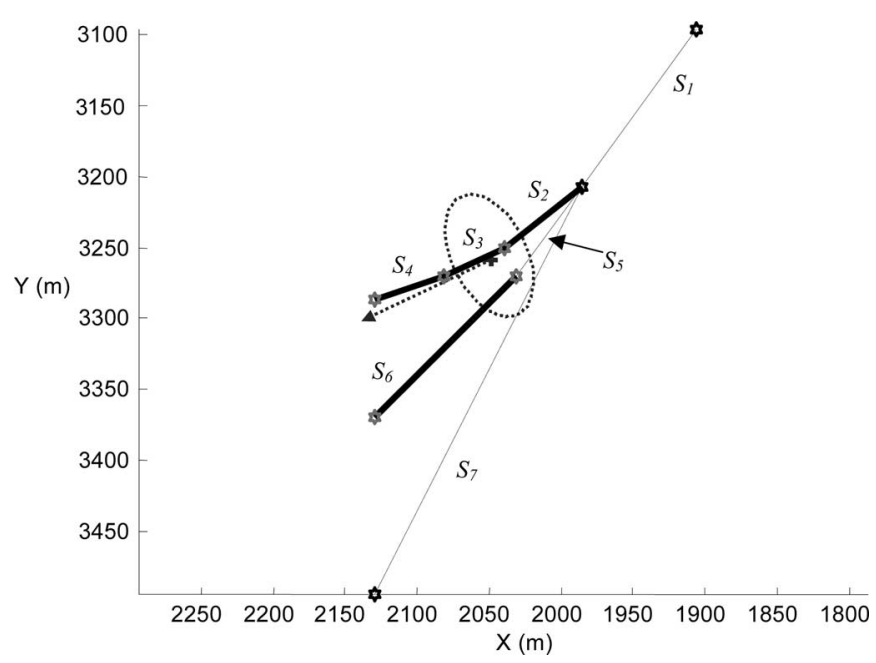

Fig. 19. Car on the exit ramp.

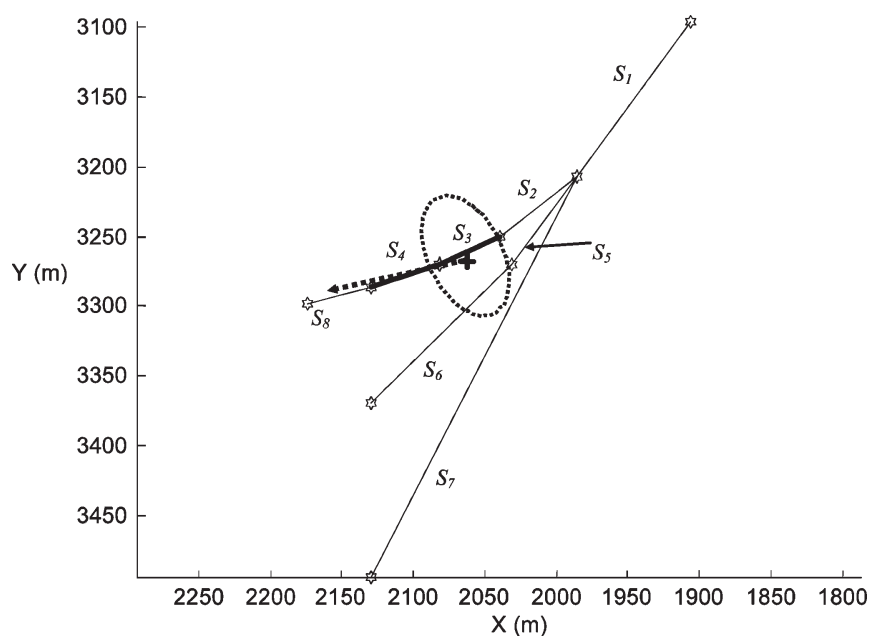

Fig. 20. Car on the exit ramp.

selection is ambiguous. The level of ambiguity depends if the selected segments make part of the same road. For example, segments $S_{3}$ and $S_{4}$ are credible at time index 96. Obviously,

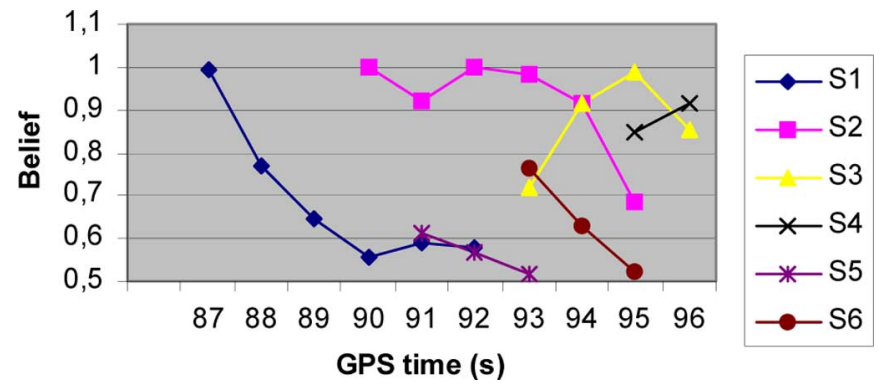

Fig. 21. Belief versus time.

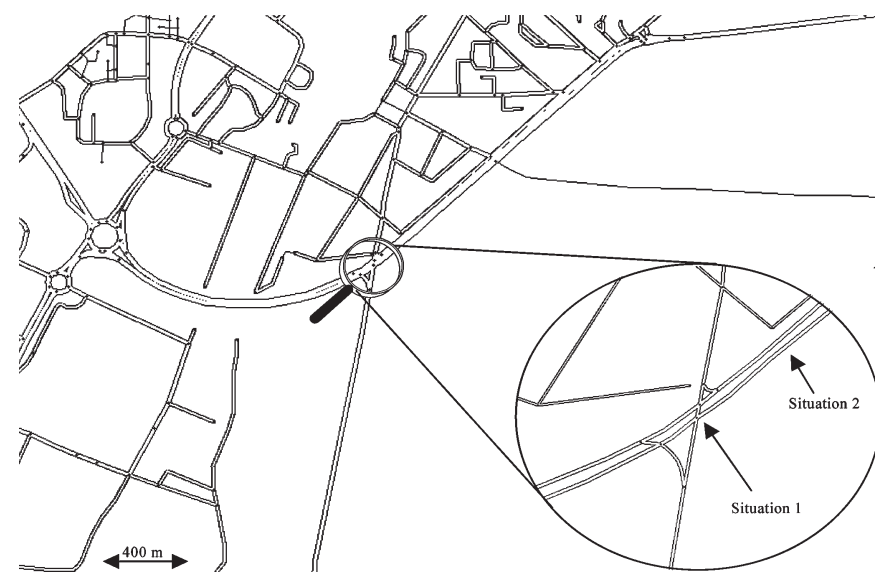

Fig. 22. Overhead view of the test trajectory.

this situation is less ambiguous than the one of time 91, during which three segments of two roads are credible.

Let us analyze the behavior of the method in another potentially ambiguous situation. In Fig. 22, two critical situations occur. The first one corresponds to a junction of three roads: Two present the same heading, whereas the third road has a $45^{\circ}$ angle. In the second situation, three roads have the same heading and are very close to each other $(<10 \mathrm{~m})$. The speed of the vehicle is about $70 \mathrm{~km} / \mathrm{h}$.

Fig. 23 shows how the system deals with the first critical situation: Several credible roads are good candidates. First, it will be remarked that only the segments that represent the parallel road are selected. Moreover, as these segments belong to two different roads, the situation is ambiguous. If the application that uses the road-matching method can tolerate errors, the most credible segment can be output. In this particular case, the most credible segment corresponds to the right road, but this is purely fortuitous.

Fig. 24 shows the result processing of the second critical situation. In this situation, the vehicle is traveling on a wide road, which is represented by two arcs. A secondary road is parallel and very close to the main road. The road selection method extracts four segments. Once again, the situation is ambiguous because the segments belong to three different roads.

Finally, we have tested the road selection algorithm on the $4.5-\mathrm{km}$ route that is presented in Section II-B (see Fig. 3). In Fig. 25, the dotted path represents the DGPS position that is provided by the Trimble receiver that is converted into the 2-D frame of the map. Each point of the second plot (plotted with "+") corresponds to the nearest point of the most credible 

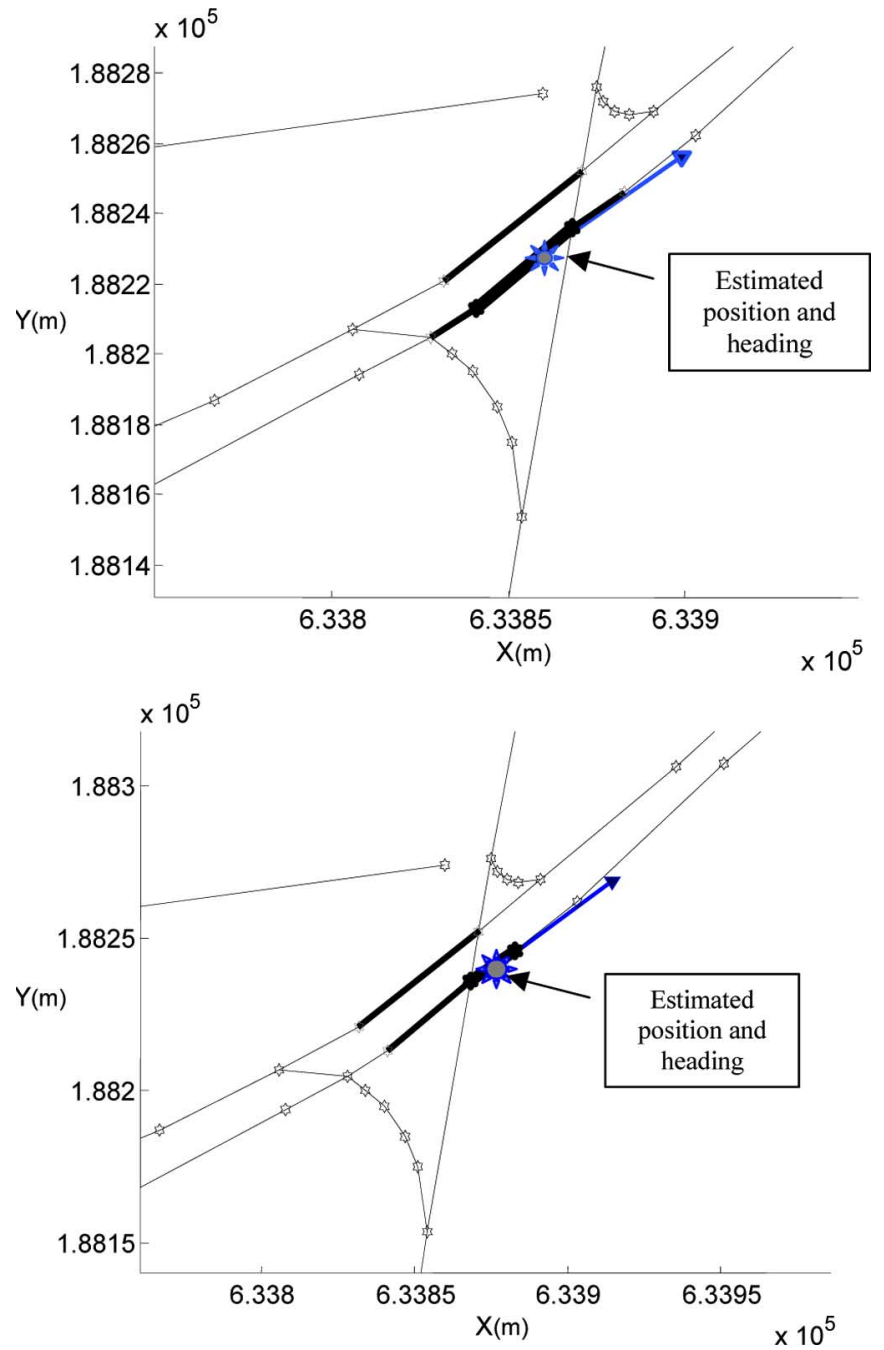

Fig. 23. Credible segments are in bold, and the most credible is in large bold (French Lambert coordinates).

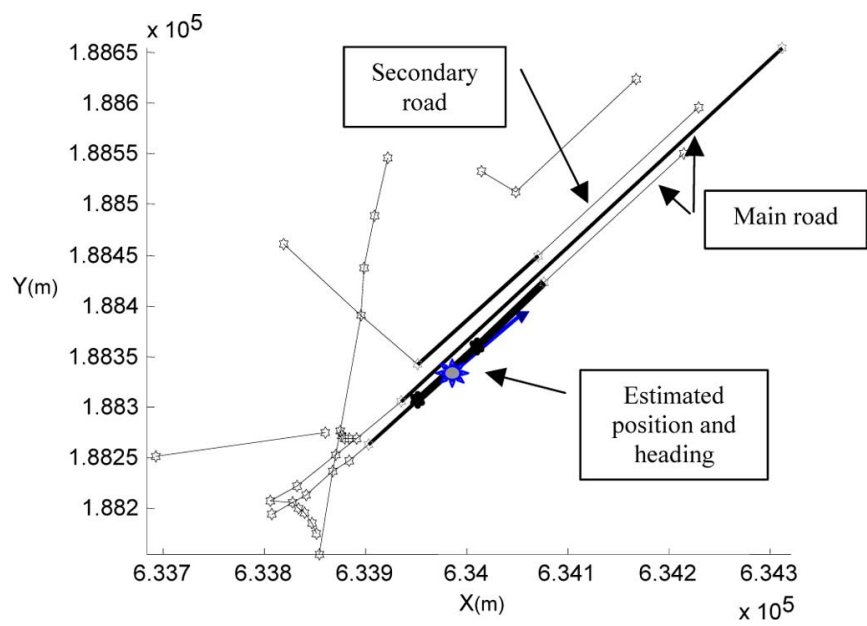

Fig. 24. Credible segments are in bold, and the most credible is in large bold (French Lambert coordinates).

segment to the estimated position that is provided by the EKF fusion of GPS and odometry. The origin of Fig. 25 has been translated to facilitate the readability of the scales of the axes. Even if all the credible segments are not indicated in this

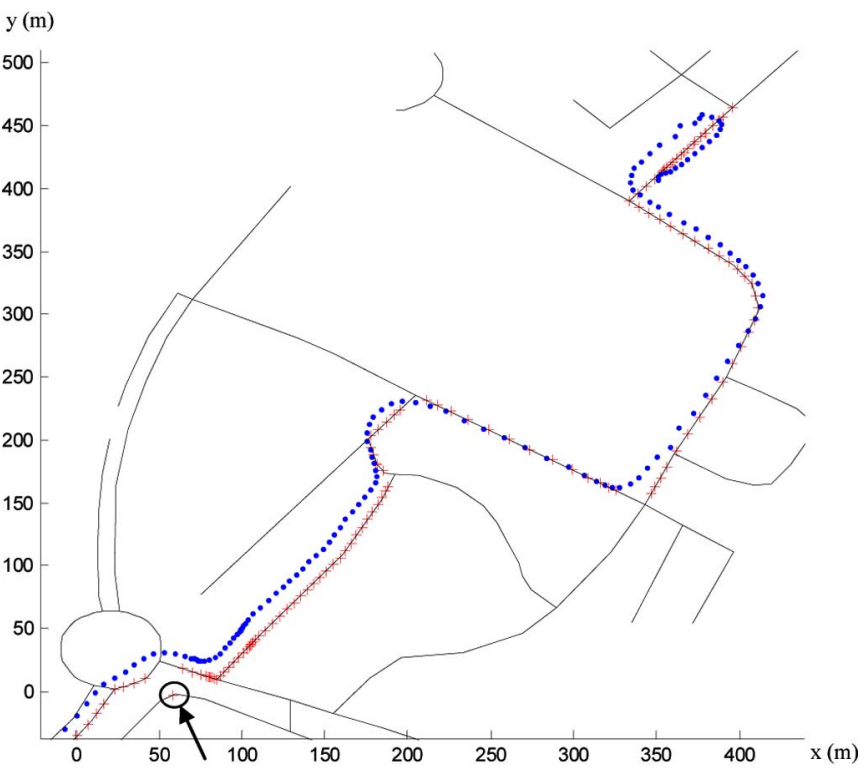

Fig. 25. GPS points $(\cdot)$ and nearest points of the candidate roads $(+)$.

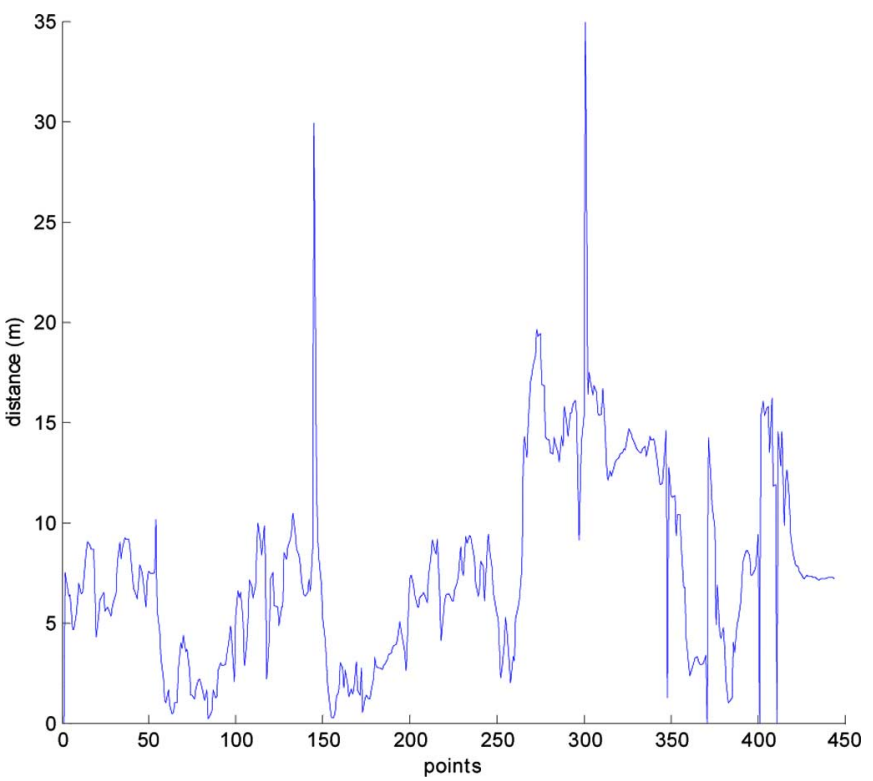

Fig. 26. Distance between DGPS path and candidate roads.

figure, it has been verified that the right segment is always on the list of the credible segments that are provided by the road selection method. One can notice that sometimes, the most credible segment can be incorrect, particularly in roundabouts, like the point surrounded by a circle. Since the road selection method is static (i.e., not recurrent), an incorrect selection has no effect on the next selection stage. This indicates robustness to fault matching if the most credible segment is considered as the matched segment. Nevertheless, we think that in case of ambiguity (i.e., the selected segments belong to different roads), a multihypothesis matching is preferable.

In Fig. 26, the distance from the DGPS position to the most credible road is plotted. It illustrates the good performance of the road selection method since this distance is on the order of several meters. We think that this is mainly due to an offset between the map and the GPS navigation solution, which is 
clearly visible in the figure. The two large values (index $\sim 150$ and index $\sim 300$ ) correspond to wrong selections, the second one being that of Fig. 25. It is important to notice that the success of a map-matching algorithm that is based uniquely on the selection of the most credible segment is contingent on the availability of accurate navigation solution and small offsets of the map.

\section{CONClusion}

This paper has presented a multicriteria fusion technique for the selection of roads from a road network database, which is a key issue in road matching. The main contributions of this paper are the formalization of this problem using the framework of Belief theory, the development of assignment functions (called criteria), and an experimental validation with real data using a DGPS and the ABS sensors. Two criteria have been proposed and developed. They use an estimated pose (position and heading) of the car that is obtained due to the fusion of GPS and ABS using an EKF. These criteria take into account the estimation error as well as geographical errors. It is interesting to note that in Belief theory, the lack of knowledge concerning a criterion can be quantified (in this particular case, it is the Perhaps hypothesis) and managed in the fusion process. Moreover, as different decision laws can be applied, different behaviors can be obtained. If a reliable behavior is desired, the ideal decision law needs to be used, as used in this paper.

The main advantage of this strategy is that it is possible to detect an ambiguous situation, where different sources of error have led to several roads becoming indistinguishable. This method can, however, detect the fact that the vehicle is not on a road stored in the database. This situation can be encountered if the roadmap is not exhaustive.

This approach is flexible and modular in the sense that it can easily integrate other criteria. The result of the fusion of two criteria can be fused with the masses assigned by a third criterion, and so on. It is therefore possible, using the same framework, to build and fuse other criteria testing, for example, the connectivity or the direction of the segments. This feature is interesting because adding other criteria is a way of increasing the robustness of the road selection.

As the segments are dealt with one by one, the size of the frame of discernment is small, and therefore, the method is well adapted to real-time conditions. For example, it takes, on average, $50 \mathrm{~ms}$ to process ten segments with an Intel Pentium III 700-MHz computer.

Finally, this method can be considered to be an excellent tool for improving positioning reliability since it is possible to quantify the ambiguity of a situation and, therefore, the confidence in a road-matched segment. This information is crucial for the development of fusion techniques that use the selected segments to improve the localization process.

\section{REFERENCES}

[1] E. Abbott and D. Powell, "Land-vehicle navigation using GPS," Proc. IEEE, vol. 87, no. 1, pp. 145-162, Jan. 1999.

[2] D. Bernstein and A. Kornhauser, "An introduction to map matching for personal navigation assistants," New Jersey TIDE Center, Newark, NJ, Tech. Rep., 1996.
[3] D. Bétaille and P. Bonnifait, "Road maintenance vehicles location using DGPS, map-matching and dead-reckoning: Experimental results of a smoothed EKF," in Proc. IAIN World Congr. Assoc. US ION Annu. Meeting, San Diego, CA, Jun. 2000, pp. 409-416.

[4] P. Bonnifait, P. Bouron, P. Crubille, and D. Meizel, "Data fusion of four ABS sensors and GPS for an enhanced localization of car-like vehicles," in Proc. IEEE ICRA, Seoul, Korea, Apr. 2001, pp. 1591-1602.

[5] A. P. Dempster, "Upper and lower probabilities induced by a multivalued mapping," Ann. Math. Stat., vol. 38, pp. 325-339, 1976.

[6] D. Dubois and H. Prade, "Fuzzy sets and system theory and application," in Mathematics in Science and Engineering, vol. 144. New York: Academic, 1993.

[7] P. Fabiani, "Représentation Dynamique de l'Incertain et Stratégie de Prise d'Information pour un Système Autonome en Environnement Evolutif," $\mathrm{Ph}$.D. dissertation, Ecole Nationale Supérieurs de l'Aéronautique et de l'Espace, Toulouse, France, 1996.

[8] M. E. B. El Najjar and P. Bonnifait, "A road-matching method for precise vehicle localization using Kalman filtering and belief theory," $J$. Autonomous Robots, vol. 19, no. 2, pp. 173-191, Sep. 2005.

[9] J. Greenfeld, "Matching GPS observations to locations on a digital map," in Proc. 81th Annu. Meeting Transp. Res. Board, Washington, DC, 2002.

[10] F. Gustafsson, N. Bergman, and U. Forsell, "Particles filters for positioning, navigation and tracking," IEEE Trans. Signal Process.-Special Issue on Monte Carlo Methods Statistical Signal Processing, vol. 50, no. 2, pp. 425-437, Feb. 2002.

[11] R. Joshi, "Novel metrics for map-matching in in-vehicle navigation system," in Proc. IEEE Intell. Veh. Symp., 2002, pp. 36-43.

[12] M. Kais, P. Bonnifait, D. Bétaille, and F. Peyret, "Development of looselycoupled FOG/DGPS and FOG/RTK systems for ADAS and a methodology to assess their real-time performance," in Proc. IEEE Intell. Veh. Symp., Las Vegas, NV, 2005, pp. 358-363.

[13] J. S. Kim, J. H. Lee, T. H. Kang, W. Y. Lee, and Y. G. Kim, "Node based map matching algorithm for car navigation system," in Proc. 29th ISATA Symp., Florence, Italy, 1996, vol. 10, pp. 121-126.

[14] S. Kim and J. H. Kim, "Adaptive fuzzy-network-based C-measure mapmatching algorithm for car navigation system," IEEE Trans. Ind. Electron., vol. 48, no. 2, pp. 432-441, Apr. 2001.

[15] E. J. Krakiwsky, C. B. Harris, and R. V. C. Wong, "A Kalman filter for integrating dead reckoning, map matching and GPS positioning," in Proc. IEEE Position Location Navigat. Symp., 1988, pp. 39-46.

[16] C. Pradalier and S. Sekhavat, "Localization space: A framework for localization and planning, for systems using a sensor/landmarks module," in Proc. IEEE Conf. Robot. Autom., 2002, pp. 708-713.

[17] J. S. Pyo, D. H. Shin, and T. K. Sung, "Development of a map matching method using the multiple hypothesis technique," in Proc. IEEE Conf. Intell. Transp. Syst., 2001, pp. 23-27.

[18] M. A. Quddus, W. Y. Ochieng, L. Zhao, and R. B. Noland, "A general map matching algorithm for transport telematics applications," GPS Solut., vol. 7, no. 3, pp. 157-167, 2003.

[19] C. A. Scott and C. R. Drane, "Increased accuracy of motor vehicle position estimation by utilizing map data, vehicle dynamics and other information sources," in Proc. Veh. Navigat. Inf. Syst. Conf., 1994, pp. $585-590$.

[20] G. A. Shafter, A Mathematical Theory of Evidence. Princeton, NJ: Princeton Univ. Press, 1976.

[21] S. Syed and M. E. Cannon, "Fuzzy logic based-map matching algorithm for vehicle navigation system in urban canyons," in Proc. ION Nat. Tech. Meeting, San Diego, CA, 2004, pp. 982-993.

[22] J. Tanaka, K. Hirano, T. Itoh, H. Nobuta, and S. Tsunoda, "Navigation system with map-matching method," in Proc. SAE Int. Congr. Expo., 1990 , pp. $45-50$.

[23] G. Taylor and G. Blewitt, "Road reduction filtering using GPS," in Proc. 3th AGILE Conf. Geographic Inf. Sci., Helsinki, Finland, 2000, pp. $25-27$.

[24] L. A. Zadeh, "A simple view of Dempster-Shafer theory of evidence and its implication for the rule of combination," AI Mag., vol. 7, no. 2, pp. 85-90, 1986.

[25] Y. Zhao, Vehicle Location and Navigation Systems. Norwood, MA: Artech House, 1997.

[26] L. Zhao, W. Y. Ochieng, M. A. Quddus, and R. B. Noland, "An extended Kalman filter algorithm for integrating GPS and low-cost dead reckoning system data for vehicle performance and emissions monitoring," J. Navig., vol. 56, no. 2, pp. 257-275, 2003.

[27] N. N. Zheng, S. Tang, H. Cheng, Q. Li, G. Lai, and F. Y. Wang, "Toward intelligent driver-assistance and safety warning systems," IEEE Intell. Syst., vol. 19, no. 2, pp. 8-11, Mar./Apr. 2004. 


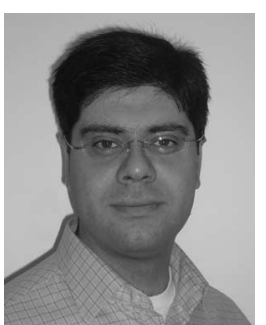

Maan El Badaoui El Najjar was born in Tripoli, Lebanon, in 1975. He received the Engineer diploma and the M.S. degree in control system and automation from the Institut National Polytechnique de Grenoble, Grenoble, France, in 1999 and 2000, respectively, and the $\mathrm{Ph} . \mathrm{D}$. degree in control systems from the Université de Technologie de Compiègne, Compiègne, France, in 2003.

$\mathrm{He}$ is a permanent Assistant Professor with the LAGIS Laboratory, UMR CNRS 8146, Ecole Centrale de Lyon, Université des Sciences et Technologies de Lille, Villeneuve d'Ascq, France. He is also a member of the MAIA project of the LORIA-INRIA Lorraine Laboratory (UMR 7503). His current research interests are in data fusion with Bayesian methods, autonomous outdoor navigation, GNSS, and 2-D/3-D GIS.

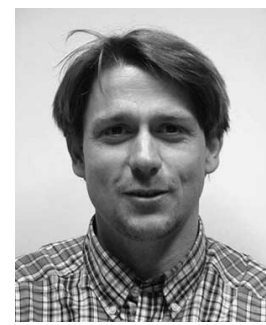

Philippe Bonnifait (M'05) was born in France in 1969. He received the degree from the Ecole Superieure d'Electronique de l'Ouest, Angers, France, in 1992, the Ph.D. degree in automatic control and computer science from the Ecole Centrale de Nantes, Nantes, France, in 1997, and the Habilitation à Diriger des Recherches degree from the Université de Technologie de Compiègne (UTC), Compiègne, France, in 2005.

He joined the Institut de Recherche en Communications et Cybernétique de Nantes (UMR 6597) in 1993. Since September 1998, he has been with the Laboratoire Heudiasyc, UMR CNRS 6599, Departement Genie Informatique, Universite de Technologie de Compiegne, where he is currently an Associate Professor. From February 2001 to June 2004, he participated to the FP5 Growth project "Roadsense," which is dedicated to the evaluation of Advanced Driving-Assistance Systems (ADAS). He has been also involved in the French project ARCOS (2001-2004) in the areas of GNSS precise positioning and advanced GIS systems. Recently, he worked on intelligent vehicle localization in urban areas for autonomous application in the framework of a French PREDIT project that is called MobiVip (2003-2006). Since February 2006, he has been participating in the subproject POsitioning, MApping and referencing systems (POMA) of an Integrated Project (IST FP6) that is called Cooperative Vehicles Infrastructure Systems (CVIS). His current research interests are in intelligent vehicles and ADAS, with particular emphasis on dynamic egolocalization that is based on multisensor fusion (GNSS, dead reckoning, and GIS). 